\title{
Designing Effective Serious Games: Opportunities and Challenges for Research
}

\author{
$\underline{\text { doi:10.3991/ijet.v5s3.1500 }}$ \\ F. Bellotti, R. Berta, and A. De Gloria \\ University of Genoa, Genoa, Italy
}

\begin{abstract}
Serious Games represent an acknowledged potential for instruction, because they are able to strongly motivate learners. They can also provide immersive environments where advanced users can practice knowledge and skills, also exploiting multimodal interaction. They can combine the effectiveness of computer processing and data storage, with high levels of attractiveness. Our work has investigated the state of the art research on SGs, starting from the cognitive aspects, that are necessary in order to root technological development and applications in sound theoretical foundations. The paper discusses some key aspects about SG design and exploitation: choice of components-off-the-shelf or from-scratch design, tools and methodologies for development or adaptation, intelligent tutoring, virtual coaches and affective learning, living worlds, game mechanics, Human-Computer Interaction.
\end{abstract}

While several SGs have been developed, still the literature stresses a lack of significant, extensive user tests. Further research is necessary to investigate in greater detail the real effectiveness of the various types of SGs. The paper proposes several research questions - that range from requirements elicitation to design and from deployment to use and evaluation - to be answered in order to avoid technology pushing and drive technological research according to the requirements of the end-users and stakeholders.

We believe that deepening the analysis about these issues is key to strengthen the foundations of SG research, for which we identify four major directions: definition of metrics and learning progress evaluation tools; methodologies and tools for designing games from various topics and for various users; computing and communication architectures; technologies that can enhance the overall system performance.

Index Terms-Serious Games, virtual reality, simulation, intelligent tutoring, human-computer interaction, cognitive load theory

\section{INTRODUCTION}

Education has become an emergency in our societies. Education involves everybody, at every age. However, it is fundamental for the young people and requires responsible adults that introduce each child or young man to the totality of the reality. Education, thus, has a very broad spectrum and cannot be guaranteed by single tools. But it is a reasonable question to investigate what Information and Communication Technologies tools can be developed and used in order to favour educators in their mission. In particular, there are some aspects of education - notably, learning and training - that seem particularly suited to computer-based support. And in this regard, some game technologies seem effective and promising, also because they seem address several e-learning problems such as high dropout rates due to frustration and the lack of motivation to continue studying $[1,2]$.

The potential of Serious games (SGs) - games that use pedagogy to infuse instruction into the game play experience $[3,4]$ - is relevant, because a large and growing population is familiar with playing games, that can present users with realistic and compelling challenges, highly stimulating their information processing capabilities and capturing their concentration span for long duration. "Games embody well-established principles and models of learning. For instance, games are effective partly because the learning takes place within a meaningful (to the game) context. What you must learn is directly related to the environment in which you learn and demonstrate it; thus, the learning is not only relevant but applied and practiced within that context (Situated cognition)". "Researchers have also pointed out that play is a primary socialization and learning mechanism common to all human cultures” [5]. [6] claims that students can only remember 10 percent of what they read, but almost 90 percent, if they engage in the job themselves, even if only as a simulation.

Good SGs challenge players sense immersive situations, providing concrete, compelling contexts where the player gets concretely involved. This is important to motivate learners and also to show the concrete relevance to everyone's life of subjects (e.g., maths and physics) that are frequently considered as cold and abstract, but whose applications to improve our understanding (and prediction) of the world and its processes are surprising and give satisfaction to students. Moreover, SGs can provide an excellent context not only to acquire and test knowledge and skills, but also to closely examine an environment without the barriers of time and space (and any other type of costs), thus can be gyms where new knowledge, practices and solutions can be developed. Play, in fact, supports players in exercising five kinds of freedom (freedom to fail, experiment, fashion identities - possibility to experience a situation from multiple perspectives [7] -, of effort and interpretation), that can complement formal learning by encouraging learners to explore various situations [8]. SGs can be multiplayer, favouring team-building and cooperation in facing issues, shaping real communities of learners. The widespread diffusion of mobile gaming is opening further perspectives also for learning and socialization (e.g., [9]).

Games provide immediate feedback, that may be efficient for procedural learning [10]. Virtual environments and simulations are effective over non-computer methods, as they allow for high levels of fidelity and an immersive 
experience [10]. This is expected to be ever more important in particular to support training, particularly in areas of training where learning fade could be critical and involve the loss of lives, such as in emergency response training [10].

Performing a survey with e-learning professionals and experts, and comparing outcomes with literature review, [11] stresses a "positive view", as serious games are perceived as "de facto effective learning environments [12] because games challenge and support players to approach, explore and overcome problems". Moreover, they "offer players the capacity to try out alternative courses of action and afford players to experience the consequences of these alternatives. They are adaptive, absorbing and immersive. They also provide feedback and assessment and allow for personalised learning. Furthermore, they place the learner in an active role, stimulating him/her to think more critically and they lend themselves to collective and social use. They challenge and support learners and implicitly motivate them".

[13] reports his experience at the DoD, where three different Components-Off-The-Shelf (COTS) management simulation videogames were added to 3 courses (first and third year of the armed forces) in a quest for new methods to "get the most from our new best and brightest". The study reports that "Students in classes using the game scored significantly higher means than classes that did not”.

However, the [11] survey highlights also some important limitations. [14] mentions that intended learning outcomes and game objectives might conflict and that games can distract from the learning content. The 'suspension of disbelief', typically required in a game, can negatively influence the learning processes. Also, certain sociodemographic groups may be excluded and 'hijacking' gaming as an educational technology might result in aversion towards the medium. Also, some game features (e.g., difficulty level, duration, aesthetic, interaction modalities) might endanger the instructional goal [12]. [15] - in a study mapping serious games and simulations into healthcare domains considering a deep structure of cognitive development from novice to expert - concludes that "there is insufficient evidence to know if extant serious games improve healthcare education but there is evidence that they may inculcate inadequate clinical pattern recognition”.

Comparing medical and aviation training and simulation, [16] states that proper simulation is very important if the training is to be effective. As shown by the huge results achieved by aviation training through simulation, simulation "can provide a safe environment that allows training and feedback on specific cognitive or psychomotor tasks far more frequently than would be the case if the trainee were waiting for actual equipment or specific conditions". However, the paper highlights that "most of the widely used simulation games have been developed for commercial reasons and including education features has typically come as an afterthought”. And stresses that research has to focus on development of instructional requirements and integration strategies for games to ensure they are able to accomplish the intended goals.

Concerns have emerged about an extensive use of videogames, in particular by the youngsters. This is to be kept in consideration also in the case of educational games, in particular when considering hyper-stimulation and distinction between virtuality and reality. This point on the one hand stresses again the necessity of responsible adults able to manage the education process and the other hand the importance of user-centered design, considering all the process stakeholders, starting from the parents.

Summarizing, there is a consensus in the scientific community about the instructional potential of games, mostly because they are considered motivating (e.g., [17, $12,18])$. Few tests have shown the educational value in particular in the business/management field. But, in general, most of the authors agree that more extensive tests are to be performed (e.g., [19, 20, 21, 22, 23, 9, 17, 24]). Some experiences highlight the importance of games for awareness raising (e.g., [24] for societal issues). However, stressing the emotive aspect, that is interesting for some types of games - in particular for advertising -, may be misleading for proper education. The concern is emerging that "we are just beginning understand when and how games promote learning, and why" [25]. In particular, there is a need for scientific and engineering methods for building games as means that provide effective learning experiences [3].

The objective of this paper is to critically analyze the state of the art of serious gaming and try to understand where and how it is effective and what directions look fundamental for future research.

The remainder of the paper is organized as it follows: section II provides an introduction to Serious Games. Section III focuses on instruction and cognition, as a theoretical basis of SG design and development. Section IV investigate several SG design and exploitation aspects that we consider key for present and future research. In section V we focus the discussion on the SandBox SG paradigm, that we consider quite effective for instruction. Section VII draws the final conclusions and suggests the lines for research.

\section{SERIOUS GAMES}

Following from the Platonic differentiation between games for fun and games for learning, the term "serious game" was firstly used by Clark Abt [26]. The book was mainly about board and card games. However, Abt's definition is still applicable in the computer age: "Reduced to its formal essence, a game is an activity among two or more independent decision-makers seeking to achieve their objectives in some limiting context. A more conventional definition would say that a game is a context with rules among adversaries trying to win objectives. We are concerned with serious games in the sense that these games have an explicit and carefully thought-out educational purpose and are not intended to be played primarily for amusement."

The "serious game" term as we intend today was firstly used in 2002, with the start of the Serious Game Initiative lead by David Rejeski and Ben Sawyer in the US, and taken up in Europe by the formation of the Serious Games movement, in particular in the UK. The SG Initiative focuses "on uses for games in exploring management and leadership challenges facing the public sector. Part of its overall charter is to help forge productive links between the electronic game industry and projects involving the 
use of games in education, training, health, and public policy"1.

SGs were initially conceived to train people for tasks in particular jobs, such as training army personnel, or training insurance salesmen. These tasks were characterized by their specificity and applicability for particular work-related purposes and are typically targeted at a captive audience.

The MIT Education Arcade document [8] discusses a number of examples of learning games, such as Zoo Scene Investigators, Palmagotchi, Racing Academy, Ayiti, Gamestar Mechanic, The Calm and the Storm, Mind Rover, Lure of the Labyrinth, the Federation of the American Scientists' Immune Attack. The document shows that uses of SGs now "span everything from advancing social causes to promoting better health to marketing. The class of games known as Games for Change $^{2}$ are being designed with a social or political agenda to get people to consider particular issues. Members of Games for Health ${ }^{3}$ design games for both patients and practitioners with a medical purpose in mind. Advergaming is a popular form of advertisement that delivers commercial messages through games”.

Prensky has put together a list of five hundred "serious" games that can be used to teach different content ${ }^{4}$, and his new book and accompanying Web site ${ }^{5}$ provide even more guidance on using games for learning.

Even the use of Commercial Off The Shelf (COTS) games is being considered for education, given their popularity, validity and cost-effectiveness with respect to developing SGs ad-hoc designed to support specific curricular activities. COTS games already being used in the classroom include Civilization (history), Age of Empires II (history), CSI (forensics and criminal justice), The Sims 2 (making complex social relationships), Rollercoaster Tycoon (Engineering and Business Management), and SimCity 4 (Civil Engineering and Government). Three business/management simulation COTS games (namely: Industry Giant II, Zapitalism and Virtual U) have been proved as useful for instruction by the experiment on business topics in formal education settings [13].

It is important to highlight that SGs do not target exclusively power-gamers (typically young males fond of First Person 3D immersive experiences) . Power-gamers represent just $11 \%$ of the gaming community, while other types of players (Social, Leisure, Dormant, Incidental and Occasional gamers) have acquired a huge weight . This audience enlargement has been enabled by new typologies of games (e.g. brain training games, casual games, virtual pet caring, intellectual challenges such as Professor Layton and the Curious Village and Phoenix Wright: Ace Attorney) and by new modalities of interactions (e.g. online collaboration, verbal commands, gesture-based control, social environments, family gaming). This is also particularly promising for games for learning, since it shows that games that are able to match the expectations and requirements are able to capture a huge number of customers . Moreover, several new games (e.g. mobile,

\footnotetext{
${ }^{1}$ http://www.seriousgames.org

http://www.games4change.org

3 http://www.gamesforhealth.org

${ }^{4}$ http://www.socialimpactgames.com

5 http://www.gamesparentsteachers.com
}

some consoles and new interaction modalities) are inexpensive to produce and run on low-cost computational platforms.

New types of gaming/leisure environments also include Virtual Worlds (VWs). The frontier with SGs is quite blurred. Both rely upon the same virtual reality technologies and give emphasis to interactivity and immersion. VWs are being used by educational institutions to support learning, in particular given the possibility of overcoming the barriers of time and space for geographically distributed users. "The success, and wide reporting, of Second Life has helped to highlight the wider use of immersive worlds for supporting a range of human activities and interactions, presenting a wealth of new opportunities for enriching how we learn, how we work and how we play” [27].

SGs and VWs technologies allow the creation of realistic 3D environments, with full sensory representation and immersion. These environments, populated by players' avatars and by Virtual Human Characters (VHCs) controlled by the computer's Artificial Intelligence (AI), can re-present the appearance, behavior, beliefs and traits of specific cultures. This is the concept of Living Worlds (LWs). VHCs live autonomously in the LW and perform goal oriented activities, interacting with each other as people do in daily life. A player can interact with such VHCs (e.g. speak, provide or receive help, exchange objects). This communication happens through the cultural mediation modalities typical of the specific represented population. Thus, these environments allow players to live experiences contextualized in a precise cultural environment. For example, the US Army has developed a training SG to educate soldiers about foreign cultures (e.g. Afghanistan) [28]. While in the Palestinian context , players act as journalists investigating facts and beliefs about the conflict.

\section{INSTRUCTION AND COGNITION}

In order to design, develop and deploy effective tools for learning, it is necessary to consider all the stakeholders (users, educators, families, reserachers, developers/industries) and the whole cycle from research to market and vice-versa. From a scientific point of view, this needs considering a complex mix of disciplines and technologies, such as: Artificial Intelligence, HumanComputer Interaction, networking, computer graphics and architecture, signal processing, web-distributed computing, neurosciences. These technologies are to be developed and exploited in a target-oriented multidisciplinary approach that puts the user benefits at the centre of the process. Given the instructional goal, SG $\mathrm{R} \& \mathrm{D}$ should be strongly grounded in proper educational foundations. In other words, "Serious games use entertainment principles, creativity, and technology to meet government or corporate training objectives, but these principles alone will not guarantee that the intended learning will occur. To be effective, serious games must incorporate sound cognitive, learning, and pedagogical principles into their design and structure” [3].

In this regard, we believe that it is important to stress the point that, while serious games are frequently seen as "de facto" instructional, the combination of entertainment and knowledge acquisition is far from being immediate. Games are easily "per se" motivating - anche can be 
succesfully employed with this aim. However, the next step towards instructional effectiveness is more difficult to accomplish.

[29] argues that "although unguided or minimally guided instructional approaches are very popular and intuitively appealing, these approaches ignore both the structures that constitute human cognitive architecture and evidence from empirical studies over the past half-century that consistently indicate that minimally guided instruction is less effective and less efficient than instructional approaches that place a strong emphasis on guidance of the student learning process. The advantage of guidance begins to recede only when learners have sufficiently high prior knowledge to provide "internal" guidance”. In particular, [29] refers to the cognitive load theory (CLT) [30], that stresses that information needs to pass through the working memory in order to be learnt and stored in long-term memory. This requires explicit teaching because of the limitations of the working memory. [29] highlights the importance that "instruction [is] based on the facts, laws, principles, and theories that make up a discipline's content" and states that "it may be an error to assume that the pedagogic content of the learning experience is identical to the methods and processes (i.e., the epistemology) of the discipline being studied and a mistake to assume that instruction should exclusively focus on application”. "Not only is unguided instruction normally less effective; there is also evidence that it may have negative results when students acquire misconceptions or incomplete or disorganized knowledge".

Analysing user experience of Crystal Island, [31] reports that "high-achieving science students tended to demonstrate greater problem-solving efficiency, reported higher levels of interest and presence in the narrative environment, and demonstrated an increased focus on information gathering and information organization gameplay activities". However, "lower-achieving microbiology students gravitated toward novel gameplay elements, such as conversations with non-player characters and the use of laboratory testing equipment”. Thus, they stress the need for "scaffolding techniques to promote effective problem solving, improved learning outcomes and sustained engagement for all students". Observing the gameplay, the authors noticed that "highachieving students tended to utilize more traditional science resources such as textbooks and worksheets while attempting to solve the presented mystery. In contrast, low-achieving students employed the help of expert nonplayer characters and virtual lab equipment to aid in their quest”. These observations seem to stress the fact that learning is a complex activity that requires graduality and needs several steps, that have to be supported by various tools (e.g., paper and digital, reading and writing, etc.) and generally have to be guided, possibly by a real adult, in order to be meaningful/compelling for the learner and not to waste time and energies.

Thus, free exploration or simulation of a potentially educational environment is not enough. The instructional process needs that the learner is guided and accompanied by a teacher/educator, so that he can get the sense, the meaning of what he is doing from the presence of a reliable adult. What computer systems (including games) can do is to support teachers - for instance by building an information rich and stimulating environment, providing a welth of data obtained by tracing the user and making comparisons, favoring personalization and feedback, supporting peer cooperation and dialogue with non-peers. Games can add by themselves a plot and mechanisms that keep the flow [32, 33] and continuously spur the player to imrpove. Some systems may even substitute the teacher, which may be useful for specialized topics and in case of cost, time and space barriers. But the reference to an educator would always be important [34, 35]. Moreover beside motivating beginners to new topics -, games look promising as a practicing tool to apply and test knowledge acquisition rather than an "all comprehensive” teaching tool.

[36] cites what they define an ecception to the CLT: "we do not need to go to school to learn to listen or speak, recognize faces, learn general problem-solving techniques, or learn about basic social interactions". In such "basic areas not taught in educational institutions, immense amounts could be learned without explicit instruction", but through "immersion in life experiences". However, these exceptions are limited to very specific cases, in the infancy. And also in these cases, however, guidance is not to be neglected. For instance, parents have a key role in preparing the environment, giving indication, loving the child, etc. It is true that an additional language can sometimes be learnt easily, also by adults, through immersion in experinces, but only if it is similar to another one already known, so that learners have sufficiently high prior knowledge to provide "internal” guidance.

CLT tells that overloading working memory inhibits learning. Fading worked examples (FWEs) have emerged from CLT as an effective strategy for lowering cognitive load by providing gradual exposure to concepts [Gray]. The paper states that developing effective FWEs requires thinking clearly about the concepts to be presented, decomposing them into their basic parts, and presenting those basic parts in terms of the multiple dimensions of the overall topic. Their "suggested use of FWEs is limited to individual constructs - discrete blocks of code [in their case of computer programming didactics]. It does not (cannot?) fully address the larger problem of program design. The belief, however, is that a deeper understanding of the design, semantics and testing of program parts will facilitate development of higher level program skills”.We can argue that FWEs could be a good paradigm for developing SGs, even if completely unexplored yet.

Generally speaking, the cognition mechanisms are still far to be well known. Learning is quite a diverse ability. Different types of learning range from implicit learning (conditioning and motoric learning) to declarative learning (verbal facts or figurative contents). Different types of learning are subserved by very different neural structures, follow different trajectories and require different learning environments to be optimally efficient. Different neuroscientific methods can be used to track learning related changes in the brain. Brain Computer Interaction can be used to monitor learning during playing and to evaluate the learners' mental state. Recently developed brain imaging techniques such as functional magnetic resonance imaging (fMRI) allow locating the areas within the human brain that change their level of activation due to learning. fMRI is extremely well suited to identify the brain areas involved in a specific task and to track learning related changes in activation [37]. 
On the one hand these techniques and mesurements are useful to study new interaction techniques between humans and computers on the other hand games could provide original experimental contexts in which to study cognitive and learning processes, so that more effective teaching situations/conditions/contexts could be suggested.

\section{SG DESIGN AND EXPLOITATION ASPECTS AND CHOICES}

In the previous section we have presented what we consider as the cognition foundations for serious gaming. In this section we review the SG design research tackling hot topics, controversial points, promising lines and best practices.

\section{A. Components-Off-The-Shelf, ad-hoc implementation or customization?}

A major point concerns the choice whether to use COTS games or develop ad-hoc games for instructional use.

We have already anticipated about the use of COTS business management games at DoD [13]. Richard Van Eck [5] argues that integrating COTS in the learning process is promising for education, since it is more costeffective than developing SGs ad-hoc designed to support specific curricular activities. The paper provides many examples of COTS games already being used in the classroom, including Civilization (history), Age of Empires II (history), CSI (forensics and criminal justice), The Sims 2 (making complex social relationships), Rollercoaster Tycoon (Engineering and Business Management), and SimCity 4 (Civil Engineering and Government). For some of them there is a clear match between their explicit content and the classroom content. For others, the match is between a course aims and skills and the underlying strategies and the game play. In any case it is important to be able to "easily augment the game with instructional activities that preserve the context (situated cognition) of the game (e.g., by extending the goals and character roles of the game into the classroom)" [5]. That is, not only should teachers know well about the game, propose specific trajectories to the students and verify effectiveness. They should also insert the gaming experience in a sound overall educational framework.

[38] highlights that "While some educators have adopted COTS games for use in the classroom, such games may not always meet the individual requirements of lecturers whose courses are tied to specific learning outcomes". Their proposal of creating SGs through crossdisciplinary team projects - their paper looks at "tensions generated between the pedagogical requirements (of lecturers), entertainment objectives (of games designers) and technical excellence (sought by developers)" - is reasonable, especially at an initial phase, where several experiments should be conducted in order to understand why, how, under what conditions and for whom and for what SGs can systematically help education. However, costs are very high and scalability issues relevant.

Indeed, popularity of SGs has raised the need that "people without professional game design skills, such as teachers, corporate trainers, therapists and advertising professionals, request tools that could allow them to create or modify such games" [39]. The authors state that SGs already exist in several domains, but "they are only designed by professional game designers", while "offering the ability to create prototypical Serious Games with simple tools to a market studying an area of knowledge but lacking game design skills should allow more diversity in "Serious Games" and bring more attention to them". As a preliminary investigation [39] have analysed "Gaming 2.0" examples, as a way for players to create videogame content without skills from the entertainment videogame industry, concluding that more specific "serious" tools should be explored for SG design.

\section{B. Tools and methodologies for development/adaptation of SGs}

Customization of games could be an interesting area for the future. In any case, efficient development of effective SGs is a huge concern for research since development costs are one of the major drawbacks of instructional tools [40]. There is now the need for tools/methodologies that enhance development of SGs [41]. Delta3D [42] is an open source GE that is being extended with facilities aimed at supporting efficient building of AI-enhanced SGs. Such extension modules include: finite state machine, waypoint-based navigation, and support to coding AI in high-level script languages. Current developments involve application of predictive models that learn from the flow of events and allow a more proactive agent behavior.

StoryTec [43] is a storytelling platform consisting of an authoring environment and a runtime engine. The platform allows keeping the story structure separated from the story content. Hence, it is possible to create and play different story peculiarities (for instance $2 \mathrm{~d}$ vs $3 \mathrm{~d}$ ) based on the same story structure and subsequently use different players to be controlled via high-level commands from the Narration Controller.

Adaptive educational games offer a motivating environment reinforced with a personalized learning experience. However, adapting a game to enhance its educational benefit may endanger its intrinsic motivation and flow. ALIGN is a generic and reusable architecture designed maximize re-use in developing adaptive SGs by focusing on provision of user motivational and hinting support and meta-cognitive feedback [44].

RETAIN is an SG design paradigm aimed at applying instructional strategies concurrent to game development [45]. It highlights the importance of the presentation and feedback of the didactic choices to the player and their linkage to reinforce the lesson and test the transfer of knowledge. This interesting approach is general may be used in several different contexts.

Research has focused on how to attach the adaptation problem also after the game development stage. [2] presents SeGAE, an author-friendly environment that offers to instructors a set of editors in order to modify the game design by defining new characters, objectives, victory conditions, authorised actions among other objects in the serious games.

Adaptivity, which is key to avoid stereotyped training scenarios and predictable game-play, is a matter also of supporting instructors in exploiting their knowledge about both players and learning goals in order to provide more effective gaming and learning experiences. [46] presents a teaching-through-SG framework to enable instructors to 
create adaptive scenarios through specification of what players have to learn (pre-game scenarios), how they should learn it (in-game adaptable objects and events) and, afterwards, what went missing (post-game scenarios).

Other tools and methodologies for development/adaptation of SGs will be presented in the next section, that considers the family of SandBox Serious Games.

\section{Intelligent tutoring}

Intelligent tutoring $[47,48]$ has proven effective for learning, but its application in the field of gaming is more difficult, also because games need to support appealing and meaningful player agency. Interactive Narrative techniques have been investigated to this end. The Interactive Storytelling Architecture for Training (ISAT) provides tailored training experiences within an interactive story experience [49]. The central component of the system is an intelligent director agent responsible for individualizing the training experience. The director reacts to trainee actions within a scenario, dynamically adapting the environment to the learning needs of trainee as well as the dramatic needs of the scene [50].

Narrative-centered learning environments are an important class of educational games that situate learning within rich story contexts [31]. [51] presents a framework for creating interactive narratives for entertainment, educational, and training purposes based on an experience manager agent, which is a generalization of a drama manager. The Experience Management Framework involves semiautonomous characters agents that show believable, realistic behaviours and are able to choose their own goals and how to execute them, giving rise to emergent narrative when interacting with humans. If a user activity makes changes in the world status that make it inconsistent with the currently executing narrative plan [52], the Automated Story Director intervenes, also giving directions to the characters agents, in order to adapt the narrative plan by selecting the most applicable contingency plan from a tree of narrative adaptations. The IN-TALE interactive practice environment is an example application of the system in the field of education, in particular to promote the practice of cognitive skills such as decision making and leadership [53].

Crystal Island is an environment that supports an inquiry-based approach, where the story is a sort of a container of elements to be taken in considerations in order for the player to solve problems in the domain of biology [54].

Thespian is an interactive drama system supporting goal-driven decision-theoretic multiple agents that are responsive to user's interaction while maintaining consistency with their roles in the story [55]. Thespian specifically allows encoding learning goals [56], so that virtual characters can assist the learner, provided he has a proper approach to the dialogue. Moreover, characters have a goal that the learner acquire skills specified by the pedagogy. A character can then use its mental model of the learner as a student model to measure the degree to which the pedagogical goals are achieved.

Emergent narrative is a type of narrative framework inspired by the role of the Game Master in non computerbased role-playing games that are seen as genres in which narrative structure and interactive freedom are often successfully reconciled [57]. Given that there is no longer a unique, pre-determined plot, the methods applied in other story managers, designed to defend such a plot against the variability introduced by interactive freedom, seem inappropriate. To this end, a story facilitator has been implemented within a character-based system using the FAtiMA agent architecture [58]. The concept of emergent narrative should not be seen as one in which characters are thrown together in the hope that narrative experience will emerge, RPGs indicate that reconciling the creative powers of interaction and the structural imperatives of the author are feasible provided plot is seen as a guide to what actually happens rather than a strait-jacket to be imposed upon participants. A Narrative Engine with emergent story properties is being developed also in the TARGET project [59]. A main motivation of the system is to provide believable, emotional agents. For this reason we are using an extension to the proven technique of BDI based agents with a modified OCC Theory of Emotions [60]. The OCC theory which sees the emotional process as based on appraisal, so that emotions are valenced (good or bad) reactions to events.

The two major approaches to interactive narrative: emergent approaches utilizing autonomous virtual character agents, and drama management approaches utilizing semi-autonomous virtual character agents were compared by [61]. The comparison is not direct, but exploits improvisational theatre as an idealized, human analogue to computational interactive narrative. Results suggest that drama management systems look advantageous when particular features are required to emerge in players' interactive experiences, thus we may consider them as more suited for instructional contexts.

\section{Virtual Coaches and affective learning}

Some SGs feature AI-driven avatars that are part of the game's narrative and are aware of the gamer/student motivation. Such motivational coach avatars exploit cognitive modelling techniques that allow taking informed decisions about the gamers' skills in a learning topic and in so doing, personalizing the interaction [62]. The use of synthetic avatars has been shown to be intrinsically motivating, helping the learner to achieve higher degrees of learning [63].

[64] describes the work-in-progress development of an interactive 3D training application generated using components of the Unreal game engine that facilitate the learning of various fundamental coaching methods via the interactive completion of a variety of interrelated tasks. The system involves six gym training units: dynamic warm-up and stretch; fun warm-up game, domes and dishes; fast foot ladder activity; balance and coordination; Hand-eye reaction using visual acuity ring; cool-down.

While gaming technologies have known tremendous improvements in computational/networking performance and the physical realism of characters and environment, the affective/psychological realism of characters and their social interactions has not reached similar levels. Achieving good results in these areas is expected to make SGs more effective and engaging as teaching and therapeutic tools. [65] outlines an ambitious set of requirements for a game engine capable of supporting the development of affective games: games capable of recognizing the players' emotions, adapting the gameplay and game character behavior to these emotions, and able to generate affectively-realistic social interactions among game characters. 
This is "an ideal set of requirements, which is currently well beyond the state of the art in affective computing and affective modeling". However, such requirements "would be divided among several constituent tools, analogously to the current division between game engines proper (providing the simulation and animation support), and the definition of the 3D objects populating the game environment”, thus defining a modular architecture able to address NonPlayer Characters (NPCs) emotional and affective realism.

SGs look to have a good potential to support affective learning. This requires recognition and interpretation of emotions and a consequent creation of emotional experiences able to motivate players and deepen learning. Moreover, there is also a need to understand affective representations and mechanisms that games support. Studying these topics, [66] propose an outline of an approach to designing games especially for affective learning, by identifying the key principles, creating a repertory of affective learning game patterns, and using methods to contextualize gameplay and facilitate learning. The authors introduce the "affective walkthrough" method for identifying affective learning during gameplay, The method can be used to support the huge amount of study yet to be performed in the area, in particular to analyse games that involve some affective learning, to "identify and document recurring design patterns that emerge". These patterns then "need to be studied to determine whether affective learning really does occur, and whether it transfers beyond the gameworld".

FAtiMA [58] is an outstanding example of an agent architecture used for synthetic agents in which emotions and personality take a central role in influencing behaviour and thus achieving more believable agents. In FAtiMA emotions are based on the OCC cognitive theory of emotions [60]. FAtiMA has been extended in the direction of an architecture modeling culturally-specific agent behaviour, ORIENT [23], as we will see in the next sub-sction.

Affect sensitivity is of high importance also for a robot companion to be able to display socially intelligent behaviour, which is a key requirement for sustaining long-term interactions with humans. [67] presents a naturalistic scenario in which children play chess with the Philips Research iCat robot companion. A person-independent, Bayesian approach to detect the user's engagement with the iCat robot is presented. The framework models both causes and effects of engagement: features related to the user's non-verbal behaviour, the task and the companion's affective reactions are identified to predict the children's level of engagement. Experimental results show that the approach based on multimodal integration of task and social interaction-based features outperforms those based solely on non-verbal behaviour or contextual information.

[22] presents a VR baby-feeding training simulator. The study is interesting to show the potential for "human simulation", even if they highlight the lack of addressing key factors in particular about the personality of the baby. "A baby may for instance be more inclined to be fed by a parent than a new babysitter. Babies at various ages and from different cultures interact with adults at various levels". Moreover, it should be stressed that humans have freedom and reducing it to computer simulation may be useful in several cases (in particular for statistics) but is not able to represent the complexity of the human reality, in particular when considering relationships between single persons.

\section{E. Living Worlds}

Living Worlds (LWs) are VWs populated with NPCs that live autonomously and perform goal oriented activities, interacting with each other as people do in daily life. These environments are very promising as settings for educational activities, in particular for the cases in which immersion in real-life situations is particularly appropriate, such as for learning a language, or how to interact with people of different cultures or attitudes (e.g. a criminal gang).

Mainly because of the globalization phenomena, people culture is emerging as a critical area of study. Serious games, particularly those associated with cognitively complex LWs, offer a promising area of research and create new thresholds for visual, auditory, behavioral, and emotional representations [28]. The authors have developed a complex LW, set in Afghanistan, with a virtual cultural trainer such as the 3D Asymmetric Domain Analysis and Training (3D ADAT) model, a recursive platform for the development and visualization of dynamic sociocultural models. This model integrates visualization, sound design, and behavioral and cultural modeling with recursive assessment tools to create a living world that is sensory and culturally realistic, and can capture cultural nuance on both the macro and micro level. The authors argue that with the correct content, the platform could introduce players to serious game representations of any framework, be it global, national, corporate, or social.

[68] presents Tactical Iraqi, a videogame that is designed to accelerate a learner's acquisition of spoken Arabic to assist in the rapid deployment of soldiers into volatile tactical situations, and Virtual Iraq, a virtual reality simulation intended to lessen the effects of Post-Traumatic Stress Disorder among combat veterans. The authors argue that these VR worlds enable participants to develop forms of cultural literacy that soldiers would otherwise be lacking.

US military personnel engaged in civil affairs and peacekeeping operations that are making extensive use of game-base situated cognition courses prior to overseas deployments report that the linguistic and cultural competence that they gained from the courses had a significant impact on the effectiveness of their operations [69]. For instance, a US Marine unit recently attributed its success (completion of a tour of duty in Iraq without a single combat casualty) to its enhanced ability to develop relationships and establish rapport with the local people. The basis of the approach relies on the idea that "intercultural competence involves awareness of the differences between cultures, knowledge about the beliefs, values, and practices of other cultures, and the skill to apply that knowledge effortlessly and effectively in interpersonal interactions". In their approach - which is common to other military applications [68, 28] -, "videogame technology is used to create virtual worlds populated by nonplayer characters that speak and understand the target language, and behave in accordance with the norms of the culture. Conversational artificial intelligence technology enables learners to engage in spoken conversations with the non-player characters. Learners must speak the target language, and behave in a culturally proficient manner, in order to succeed at the game” [69]. 
ORIENT [23] is an agent architecture based on the FAtiMA affective agent architecture extended to model culturally specific agent behavior. The motivation was the implementation of a believable educational application aimed at developing inter-cultural empathy for 13-14 year old students. The work exploited literature on cultural taxonomy and adaptation to other cultures as well as work showing that users are sensitive to the perceived culture of believable interactive characters.

Similarly, [70] present an agent architecture based on cultural factors as "they are a crucial aspect of human societies". Studying the complexity of the term "culture", the authors have pointed out some common and relevant components that distinguish cultures: rituals and values. Their work defined the concept of ritual, "a symbolic social activity that is carried out in a predetermined fashion”, and integrated it into the FAtiMA agent architecture for synthetic characters. The concept of ritual is modelled in the architecture as a special type of goal with a predefined plan. An experiment was then conducted using these two scenarios in order to evaluate if users could identify different cultural behaviour in the two groups of characters, that only differed in their rituals. The results show that users do indeed identify the differences in the two cultures and most importantly that they ascribe the differences to cultural factors.

The above are technologically outstanding examples of complex environments that have been used in training and education. Again, we stress the necessity of integrating smart guidance within the immersive experience that these VR environments can guarantee. This requires intelligent tutoring (possibly by a human teacher assisted by the system) and game mechanics that are appropriate for instruction. And understanding what mechanics are good in which context.

\section{F. Game mechanics}

Despite the abundance of literature on SGs, few papers provide a detailed description of the specific SG mechanics (and underlying methodologies) through which a topic is "translated" into a SG. Still, "many educational games do not properly translate knowledge, facts, and lessons into the language of games. This results in games that are often neither engaging nor educational” [71]. The authors suggest that design should combine "the fantasy elements and game play conventions of the real-time strategy (RTS) genre with numbers, resources and situations based on research about a real-world topic", such as energy, agriculture, etc. In this way the player should be able to learn simply by "trying to overcome the game's challenges". This is a clear and simple, general indication, but the reference to RTSs seems especially suited to managerial simulations. [71] support "procedural rhetoric", a framework for designing educational games [72]. "Drawing analogies to verbal and visual rhetoric, Bogost defines procedural rhetoric as "the practice of using processes persuasively" [73]. Using this framework, the game designer does not just create an interactive simulation for the player, but specifically infuses it with gameplay rules (i.e. procedures) that form a compelling argument. The rules could be designed to present a particular viewpoint or they could simply model known and widely accepted facts. Using the latter approach, a game that models the realworld energy economy could thus be used to make an argument about energy production and use and its effect on the environment. Players would then learn about these issues simply by playing the game" [71]. We could argue whether this approach leads to learning or (emotively) creating awareness - in particular considering the first approach, which stresses a viewpoint from which to present issues (in particular for encouraging "behavior change" - e.g., [82, 24, 83]). We certainly agree that "the real world is ripe with problems and situations that could easily inspire game mechanics that are interesting for their own sake. For example, a game about building and managing a transportation system for a city could allow players to explore different modes of transportation while trying to balance costs related to construction and maintenance of the infrastructure with congestion, pollution and fuel efficiency" and that the foundation that "using fun as the catalyst for the learning process is solid". However, translating facts and characteristics in games for effective learning is complex and represents a still largely unexplored educational research area, at least for non businessmanagement disciplines.

CancerSpace is a game format that incorporates aspects of e-learning, adult-learning theory, and behaviorism theory to support learning, promote knowledge retention, and encourage behavior change [82]. CancerSpace's design encourages self-directed learning by presenting the players with real-world situations about which they must make decisions similar to those they would make in clinics. The targeted users are professionals working in community health centers. The authors stress the main peculiarities of the game with respect to a mere collaborative 3D elearning environment. The first point is about roleplaying: the user has to help the clinical staff evaluate the clinical literature, integrate the evidence into their clinical decision-making, plan changes to cancer-screening delivery, and accrue points correlating to increased cancerscreening rates. The user takes decisions and observes whether the chosen course of action improves the cancer screening rates.

The game includes a small number (four) of patientprovider interactions in which the decider must talk with a patient reluctant to get screened, try to educate that patient, and hopefully get him or her screened. The Decider must negotiate cultural and language barriers as well as the patient's changing attitudes toward screening. The player's conversation choices are evaluated in preprogrammed decision trees, leading to success (the patient decides to get screened) or failure.

Chance is considered an important entertainment and variability feature. To this end, wildcards are implemented, that reflect certain unplanned events common to community health centers such as a budget cut, funding of a grant, or a staff member transferring to another clinic.

Other critical factors for CancerSpace concern the use of audio elements such as buzzers, chimes, and other sound effects, combined with video elements such as quick animations indicating an incorrect answer and a mentor character showing emotions ranging from strong disapproval to strong approval.

To stimulate gameplay, CancerSpace has adapted an award system, that motivates players to increase screening rates. The CancerSpace scenarios in which the decider guides the virtual clinical staff are based on researchtested interventions and best practices. Users receive points on the basis of their performance. At each game's 
conclusion, a summary screen indicates which decisions the player implemented and their effect on the clinic's screening rate.

In their LW for cultural training in Afghanistan, [28] stress several mechanics. First and foremost, the main objective: the player wins by successfully interpreting the environment and achieving the desired living-world attitude toward him. The entire living-world game space is fueled by the knowledge-engineering process that translates the essential elements of the culture into programmable behaviors and artifacts. For instance, "In Afghan culture, older men have great influence over younger men, women, and children through local traditions and Islamic law". "Ideologically, the guiding principles of Afghan culture are a sense of familial and tribal honor, gender segregation, and indirect communication”. All the NPCs in the game are modeled accordingly. These models control activities ranging from gossiping to daily behavior such as traffic/errands. The overall social environment is modeled as well (e.g., simulating generation of factions and shuras). Winning in the game "simply" requires successfully navigating cultural moves in the game space thus achieving a good overall attitude of the village toward the player.

NPCs are highly realistic both in their aspect and in their movements. Sound is designed keeping into account three dimensions: global (e.g., weather conditions and distance traffic), regional (e.g., a radio in the room) and local (e.g., footsteps, voices).

Another key aspect is serious-ness about assessment. The underlying 3D ADAT model, that allows for analysis of the cultural behavior exhibited by the player in the game. Conversations and interactions between the NPCs and the player are recorded through a text log to provide game performance analysis. The assessment tool lists all possible choices for player behavior and conversation, highlighting both the player's choice and the most culturally appropriate response. The tool provides scores on the opinion of the player at the NPC, faction, and village level. Additional comments can be provided that highlight the player's weaknesses, explaining why a particular response is most appropriate. Feedback is thus provided to improve future performance.

Describing lessons learnt about what makes games useful from a therapeutic point of view, [83], that designed and user tested stroke rehabilitation games, highlights the importance of the following attributes: ensure playability for a broad range of patients: multimodal input, provide examples, direct and natural mappings; ensure games are valuable from a therapeutic perspective: ensure that users' motions cover their full range, detect compensatory motion, allow coordinated motions, let therapists determine difficulty; ensure that games fun and challenging: audio and visuals are important, automatic difficulty adjustments provide adequate challenge, Non-Player Characters and storylines are intriguing. Future steps concern creation of connections with friends and family members, and support of socialization.

As we have seen, playing games can be cognitively demanding, thus making it difficult, especially for beginners, to distinguish between relevant and irrelevant information. Moreover, some games could be "eccessively compelling”, thus preventing a player from correctly concentrating on the instructional material or procedures to be learnt. [84] have addressed this issue by trying to subtly cue the attention of the player to the relevant material. The authors have empirically tested the use of auditory cues to guide the player in learning the correct procedure in a 3D serious triage training game. Learning did not significantly improve in the auditory cueing condition and mental model construction was even significantly worse in the cueing condition, as compared to a control group with no cues. The tests revealed that "the participants probably hardly noticed or understood the auditory cues. This could indicate that in cases where the player has to learn a certain technique, the more explicit visual cues as used in contemporary videogames, while realism-breaking, may in fact be necessary in order to have the player notice them".

[85] presents an interesting new SG approach that can be applied to general algorithm learning and visualization. Algorithm Visualization using Serious Games (AVuSG) visualizes an algorithm to be learned in four forms: a text, a owchart, a game demo, and a game. The AVuSG method also integrates learning theories and models in addition to motivation theory (namely: Bloom, Gagnè and Constructivism) to simplify algorithm learning. The overall framework is intended to be used, with different roles, by both instructors and students.

Another key aspect for gaming and learning is online collaboration, that can involve learners and educators, increasing availability and reducing barriers due to space and time [86]. Collaboration is a key skill for current jobs and practicing collaboration in concrete simulation tasks is expected to be highly beneficial (e.g., [35]).

\section{G. HCI}

Human-Computer Interaction is a fundamental aspect, in particular for computing systems that support typically human activities, such as instruction. Successful videogames have always been characterized by high-quality 2D or, now ever more, 3D graphics. This feature has made them very appealing to a wide slice of population and has to be kept also by educational games, if they want to be attractive.

However, the HCI of a system must be functional to the users and overall targets (instructional, in our case). Thus, the HCI should be designed in order to maximize the expected benefits for the users. [87], starting studies that indicate "that creating serious games with player immersion as the single driving design goal is an imprecise method for targeting specific learning objectives", provides a framework for aligning the visual style of a SG with the expected learning outcome. The proposed framework, that aligns visual design style with the six levels of cognition described in Bloom's original taxonomy, is under testing.

VR environments are very powerful for simulations, as they can guarantee immersivity. But realism for educational targets requires that also the input and output modalities are highly corresponding to the reality. This stresses the importance of Natural Language Processing (NLP) algorithms and of high-precision haptic devices with feedback forces to render the haptic feedback, which is crucial, for instance in surgical education [19].

In general, since videogames usually require constant interaction, usability issues are key in design. [88] proposed a set of heuristics by analyzing PC game reviews 
and identifying twelve common classes of usability problems. A preliminary evaluation of the heuristics suggests that they help identify and avoid game-specific usability problems. Similar usability have been proposed in [89].

Another well known issue is accessibility, whose relevance for education is apparent [90]. Concerning games [91] has introduced the concept of universally accessible games.

\section{SANDBOX SERIOUS GAMES}

The VW in which a game is set can provide itself an important value for instruction. [92] stresses the "huge potential for serious games within geography", since game can foster associative learning and presence and place may help this in contexts where. The possible scope is even broader than only geography. The advantage of associating units of knowledge with landmarks in an environment (e.g., a public building) is well known and exploited in the "method of loci", or "art of memory", in classical rhetoric [68]. To memorize a speech, the orator breaks "the text into pieces, each of which is symbolized by vividly imagined symbols or signs. In the mind's eye, the orator places each of these images into the loci” (the loci are places in the environment/building that the orator has visited sequentially and can remember very well). "Objects of discourse can then be recalled in a precise order by imagining walking through the building again, visiting each of the loci sequentially, and viewing each of the images that was placed in the loci, thereby recalling each piece of the speech in the proper order [93, 94, 95, 96]". This method "can also be applied to 3D graphical worlds with immersive multi-sensory environments", as [68] has shown in her work on "Virtual Iraq", concluding that "the common experience of walking or driving slowly, however, is uniquely well-designed for recognition and reintegration of specific memory cues”.

Actually, several effective SandBox Serious Games (SBSG) - they also have a counterpart in successful pure entertainment games, such as Grand Theft Auto ${ }^{6}$ and Oblivion $^{7}$ [97] - have an organization that that tends to support players in building a suited knowledge structure for the addressed topic(s) [19]. In rough terms, the model consists of: (1) a concrete spatial organization - the VW where knowledge is distributed (e.g., human body [98], hospital [99], Ocean [100]) and that induces spatial information processing and provides landmarks to support orientation [101]; (2) contextualized tasks, that are spread and contextualized in the VW. Tasks embody units of knowledge that can be discovered and accessed by the player. The Task Based Learning (TBL) theory [102, 103] stresses the importance of concrete, focused activities to construct knowledge and develop skills.

A player explores the environment accomplishing problem-centered missions that involve learning material that is organized as a sequence of small chunks - tasks - each of which has a specific, limited instructional target. Usefulness of this approach has been argued in [104] in comparison with independent minigames, and tests have shown its validity to provide satisfactory enjoyment levels, according to frequent gamers [89]. [105] highlighted the importance of stimulating learners to ask questions and provide context-sensitive, timely, accurate answers.

\footnotetext{
${ }^{6}$ http://www.rockstargames.com/IV/
}

7 http://www.elderscrolls.com/home/home.php
They developed a generic inquiry management tool, named MyLA (My Learning Assistant), that exploits a multimedia database to ask questions and provide more information to players during their actual VW exploration or game adventure.

Missions' complexity grows up gradually towards ever more complex objectives. The entry level itself provides opportunities for learners to work immediately on meaningful, realistic problems. This prevents the need for textual training that may be considered as boring by several users [106].

Learning scenarios are presented with the aim to maintain the performance of learners in a "narrow zone" between too easy and too difficult [3, 107], the Zone of Proximal Development (ZPD) [109].

Tasks may be instances of templates (e.g. various sorts of minigames with different contents [109]), with the advantage that interaction modalities can be re-employed by the player in several contexts, keeping the cognitive load low. Moreover, development of tasks can be efficiently supported by an authoring toolkit, allowing teachers, and even students, to develop/customize content [109].

Availability of a large database of semantically annotated tasks, that can be distributed in meaningful landmarks in the VW, leads to the possibility of implementing games that rely on the dynamic scheduling of tasks. [20] presents an Experience Engine (EE), that exploits computational intelligence algorithms to schedule tasks matching the needs estimated by profiling the user performance and with the aim of keeping the flow. The EE

In the proposed approach, a game does not need a detailed story specification. There is a high-level challenge (e.g., a treasure hunt), that spurs players to competition/cooperation through explorations of a VW. This simple narrative structure would cause a minor cognitive load to the player, leaving more space for knowledge acquisition.

Considering content production, this approach would imply a shift of focus, from narrative to the actual educational contents (the tasks), that could be easily produced also by teachers [109]. This is promising to provide users with high quality, content-rich multimedia educational experiences.

There is the risk that the player may feel a "clunky" experience, since the author is not required to specify a complete narrative. However, SandBoxGames are successful on the market (e.g., Grand Theft Auto and Oblivion), where the player builds his own narrative experience by interacting with contextualized situations and miniadventures during a geographic exploration of a wide environment. The game author specifies such situations and the game's general rewards/competition mechanisms, not the details of the plot.

\section{CONCLUSIONS}

Serious Games represent an acknowledged potential for instruction, because they are able to strongly motivate learners, in particular to create awareness about a topic. They can also provide immersive environments where advanced users can practice knowledge and skills, also exploiting multimodal interaction. They can combine the effectiveness of computer processing and data storage 
(e.g., for personalized teaching), with high levels of attractiveness.

While several SGs have been developed, still the literature lacks of significant, extensive user tests. Collecting qualitative and quantitative feedback from already implemented system is a necessity largely highlighted in literature (e.g., [19, 20, 21, 22, 23, 9, 17, 24]).

Further research is necessary to investigate in greater detail the real effectiveness of the various types of SGs. There are several questions that require an answer, in order to avoid technology pushing and drive technological research in the most suited and useful directions for endusers and stakeholders. Are SGs good for education or only for training? For what instructional objectives is an SG useful? Why? For whom (specific types of users, e.g., in terms of learning styles)? Are there some disciplines that lend themselves better to SG? How can collaborative/social learning be supported? For what subjects? How should it be used? For how long? In which contexts? With what mediation? How can it be ensured that users are fully aware of the distinction between real and virtual worlds? How can it be ensured that "trainees have a serious attitude and understand that a failure in the game could be an unforgivably serious mistake in reality" [19]? What are the obstacles to using SGs? What are the facilitators? What are the advantages/disadvantages? What types of skills are solicited, promoted or penalized by different SG approaches? What is the relevance of the social context? How can an effective dialogue between students and teachers be supported? How can students and educators use SGs for a balanced growth of the person? How can teachers be trained and supported in the use of the new tools?

We believe that deepening the analysis about these issues - that range from requirements elicitation to design and from deployment to use and evaluation - is key to strengthen the foundations of SG research, for which we identify four major directions:

- Definition of metrics and evaluation tools (in particular concerning the tracking of the actual learning progress) to be employed for an objective assessment of games

- Methodologies and tools for designing games from various topics and for various users. Practices, methods and metrics to integrate games as tools for formal teaching

- Computing and communication architectures (e.g., enhancing the current engines/agent architectures for managing personalization and experience, narrative, affectivity and emotions, assessment engines

- Technologies that can enhance the overall system performance (e.g. modeling and simulation, Virtual Reality, Human-Computer Interaction, data mining, machine learning)

\section{REFERENCES}

[1] Prensky, M., Digital game-based learning. New York: McGrawHill. 2000

[2] Yessad A., Labat J-M, Kermorvant F., "SeGAE: A Serious Game Authoring Environment", icalt, 10th IEEE International Conference on Advanced Learning Technologies, 2010

[3] Greitzer F.L., Kuchar O.A., and Huston K., "Cognitive Science Implications for Enhancing Training Effectiveness in a Serious
Gaming Context", ACM J. Educational Resources in Computing, vol. 7, no. 3, August 2007.

[4] Gee, J.P. What video games have to teach us about learning and literacy. Palgrave MacMillan: New York. 2007

[5] Van Eck R., Digital Game-Based Learning: It's Not Just the Digital Natives Who Are Restless, EDUCAUSE Review, vol. 41, no. 2, 2006.

[6] Menn, D., Multimedia in education. PC World, 1993

[7] LoPiccolo, P. We could be heroes. Computer Graphics World, 28(3), 2005.

[8] Klopfer E, Osterweil S., and Salen K., Moving Learning Games Forward, Obstacles Opportunities \& Openness, The Education Arcade, Massachusetts Institute of Technology, 2009, available online at: http://education.mit.edu/papers/MovingLearningGamesFor ward EdArcade.pdf.

[9] Tian, F., Lv, F., Wang, J., Wang, H., Luo, W., Kam, M., Setlur, V., Dai, G., and Canny, J. , Let's play chinese characters: mobile learning approaches via culturally inspired group games. In Proceedings of the 28th international Conference on Human Factors in Computing Systems, Atlanta, Ga, USA, April , 2010

[10] Jarvis, S., de Freitas, S., Evaluation of a Serious Game to support Triage Training: In-game Feedback and its effect on Learning Transfer. Proceedings of 2009 IEEE Conference in Games and Virtual Worlds for Serious Applications, 2009

[11] De Grove, F., Mechant, P., Van Looy, J, Uncharted waters?: exploring experts' opinions on the opportunities and limitations of serious games for foreign language learning. In Proceedings of the 3rd international Conference on Fun and Games, Leuven, Belgium, September, 2010.

[12] Mitchell, A., \& Savall-Smith, C., The use of computer and video games for learning. A review of the literature. London: The learning and skills development agency. 2004, available at:

[13] Blunt, R., Does game-based learning work? Results from three recent studies. Paper presented at the Joint ADLICo Lab Implementation Fest, Orlando FL, USA, August, 2008.

[14] Clark D., Learning by playing: can computer games and simulations support teaching and learning for post-16 learners in formal, workplace and informal learning contexts? Computer games in education and training. Presentation at LSDA seminar London, November 2003.

[15] Tashiro J., What really works in serious games for healthcare education, Conference on Future Play on @ GDC Canada, Vancouver, British Columbia, Canada, 2009.

[16] Kincaid, J.P., Westerlund, K.K., "Simulation in education and training," Proceedings of the 2009 Winter Simulation Conference (WSC), Dec. 2009. doi:10.1109/WSC.2009.5429337

[17] Iacovides, I., Exploring the link between player involvement and learning within digital games. In Proceedings of the 23rd British HCI Group Annual Conference on People and Computers: Celebrating People and Technology, Cambridge, UK, September, 2009.

[18] Kirriemuir, J., \& McFarlane, A., Literature Review in Games and Learning. Futurelab series, Bristol, 2004. Available online at http://www.futurelab.org.uk/resources/documents/lit_reviews/Ga mes_Review.pdfEnhanced

[19] Qin J., Chui Y., Pang W., Choi K., Heng P. , "Learning Blood Management in Orthopedic Surgery through Gameplay," Computer Graphics and Applications, IEEE , vol.30, no.2, MarchApril, 2010.

[20] Bellotti F., Berta R., De Gloria A., Primavera L., "Adaptive Experience Engine for Serious Games", IEEE Transactions on Computational Intelligence and AI in Games, Vol 1, No. 4, 2009.

[21] Pannese L, Carlesi M, Games and learning come together to maximise effectiveness: The challenge of bridging the gap, British Journal of Educational Technology, Volume 38, Issue 3, May 2007.

[22] Petrasova, A., Czanner, G., Happa, J., Czanner, S., Wolke, D., and Chalmers, A., Assessing a virtual baby feeding training system. In Proceedings of the 7th international Conference on Computer Graphics, Virtual Reality, Visualisation and interaction in Africa, Franschhoek, South Africa, June, 2010.

[23] Aylett R., Vannini N., Andre E., Paiva A., Enz S., and Hall L.. But that was in another country: agents and intercultural empathy. In 
AAMAS '09: Proc. Of The 8th International Conference on Autonomous Agents and Multiagent Systems, Richland, SC, 2009.

[24] Rebolledo-Mendez, G., Avramides, K., de Freitas, S., and Memarzia, K. 2009. Societal impact of a serious game on raising public awareness: the case of FloodSim. In Proceedings of the 2009 ACM SIGGRAPH Symposium on Video Games, New Orleans, August, 2009. doi:10.1145/1581073.1581076

[25] Johnson, W. L., "Lessons learned from games for education”, in Proc. ACM SIGGRAPH 2005 Educators program, Los Angeles, Ca, USA, 2005.

[26] Abt C., Serious Games, New York, Viking Press, 1970.

[27] de Freitas S., Serious Virtual Worlds. A scoping study. 2008. Available online at: http://www.jisc.ac.uk/media/documents/ publications/seriousvirtualworldsv1.pdf Enhanced Learning Unlimited (ELU) project. Retrieved March, 2008, from http://www.elu-project.com.

[28] Zielke M. A., Evans M. J., Dufour F., Christopher T. V., Donahue J. K., Johnson P., Jennings E. B., Friedman B. S., Ounekeo P. L., Flores R., "Serious Games for Immersive Cultural Training: Creating a Living World," IEEE Computer Graphics and Applications, vol. 29, no. 2, pp. 49-60, Mar./Apr. 2009. doi:10.1109/ MCG.2009.30

[29] Kirschner, P.A., Sweller, J., Clark, R.E. "Why minimal guidance during instruction does not work: an analysis of the failure of constructivist, discovery, problem-based, experiential, and inquirybased teaching". Educational Psychologist 41 (2): 75-86, 2006. doi:10.1207/s15326985ep4102_1

[30] Sweller, J. "Cognitive load during problem solving: Effects on learning". Cognitive Science 12 (2), 1988. doi:10.1207/s1551 6709cog1202_ 4

[31] Rowe, J. P., Shores, L. R., Mott, B. W., Lester, J. C. Individual Differences in Gameplay and Learning: A Narrative-Centered Learning Perspective. In Proceedings of the Fifth International Conference on the Foundations of Digital Games (FDG), Monterey, CA, 2010.

[32] Csikszentmihalyi M., Flow: The Psychology of Optimal Experience, New York: Harper \& Row, 1990.

[33] Sweetser P. and Wyeth P., "GameFlow: A Model for Evaluating Player Enjoyment in Games,” ACM Computers in Entertainment, vol. 3, no. 3, July 2005.

[34] Egenfeldt-Nielsen, S. Beyond Edutainment: exploring the eductional potential of computergames. University of Copenhagen, Copenhagen, DK., 2006

[35] Angehrn A., Maxwell K., Luccini M., Rajola F., Designing Effective Collaboration, Learning and Innovation Systems for Education Professionals, International Journal of Knowledge and Learning (IJKL), Vol. 5 No. 2, 2009.

[36] Sweller, J., Kirschner, P. A., \& Clark, R. E.. Why minimally guided teaching techniques do not work: A reply to commentaries. Educational Psychologist, 42(2), 115-121, 2007Enhanced Learning Unlimited (ELU) project. Retrieved March, 2008, from http://www.elu-project.com.

[37] Ischebeck A. Zamarian L, Egger K, Schocke M, Delazer M, Imaging early practice effects in arithmetic. Neuroimage 36, 2007.

[38] Rooney P., O'Rourke K.C., Burke G., MacNamee B., Igbrude C., "Cross-Disciplinary Approaches for Developing Serious Games in Higher Education," vs-games, Conference in Games and Virtual Worlds for Serious Applications, 2009. doi:10.1109/VSGAMES.2009.32

[39] Djaouti D., Alvarez J., Jessel J-P, "Can "Gaming 2.0" Help Design "Serious Games"? - A Comparative Study", SIGGRAPH 2010, Los Angeles, CA, USA, 2010.

[40] Aldrich C., A field guide to educational simulations. Learning Circuits, American Society for Training and Development. January, 2003

[41] Conconi A., Ganchev T., Kocsis O., Papadopoulos G., FernandezAranda F., and Jimenez-Murcia S., "PlayMancer: A Serious Gaming 3D Environment”, in Proc. 4th International Conference on Automated Solutions for Cross Media Content and Multi-Channel Distribution, AXMEDIS 2008, Florence, Italy, Nov. 17-19, 2008

[42] Darken R., McDowell P., and Johnson E., "Projects in VR: the Delta3D Open Source Game Engine”, IEEE Computer Graphics and Applications, vol. 25, no. 3, pp.10 - 12, May/June 2005. doi:10.1109/MCG.2005.67

[43] Göbel, S., Salvatore, L., Konrad, R. StoryTec: A Digital Storytelling Platform for the Authoring and Experiencing of Interactive and Non-linear Stories. In Nesi, P., Ng, K. and Delgado, J.: 4th International Conference on Automated Solutions for Cross Media Content and Multi-Channel Distribution, IEEE computer society, 2008

[44] Peirce N., Conlan O., and Wade V., "Adaptive Educational Games: Providing Non-invasive Personalised Learning Experiences", in Proc. of the Second IEEE International Conference on Digital Games and Intelligent Toys Based Education (DIGITEL 2008), Banff, Canada, November 2008. doi:10.1109/ DIGITEL.2008.30

[45] Gunter G., Kenny R. F., and Vick E. H., "A Case for Formal Design Paradigm for Serious Games”, CODE - Human Systems, Digital Bodies. 2006

[46] Lopes R., Scenario adaptivity in serious games, International Conference on the Foundations of Digital Games, Monterrey, Ca, 2010.

[47] Wenger, E., Artificial Intelligence and Tutoring Systems: Computational and Cognitive Approaches to the Communication of Knowledge. San Francisco: Morgan Kaufmann, 1987.

[48] Shute, V. J. and Psotka, J., Intelligent Tutoring Systems: Past, Present and Future. In D. Jonassen (ed.) Handbook of Research on Educational Communications and Technology. Scholastic Publications, 1996.

[49] Magerko B.: Adaptation in Digital Games. IEEE Computer 41(6): 87-89, 2008.

[50] Magerko, B., Stensrud, B., and Holt, L. Bringing the Schoolhouse Inside the Box - A Tool for Engaging, Individualized Training. 25th Army Science Conference, 2006. Orlando, FL

[51] Riedl M. O., Stern A., Dini D., and Alderman J.. Dynamic Experience Management in Virtual Worlds for Entertainment, Education, and Training. International Transactions on Systems Science and Applications, Special Issue on Agent Based Systems for Human Learning, vol. 4(2), 2008.

[52] Riedl M. O. and Young R. M.. From Linear Story Generation to Branching Story Graphs. IEEE Computer Graphics and Applications, 26(3), 2006. Featured article of Special Issue on Interactive Narrative.

[53] Riedl M. O. and Stern A.. Believable Agents and Intelligent Story Adaptation for Interactive Storytelling. Proceedings of the 3rd International Conference on Technologies for Interactive Digital Storytelling and Entertainment, Darmstadt, Germany, 2006. doi:10.1007/11944577_1

[54] Rowe J. P., Mcquiggan S. W., Mott B. W., Lester, J. C., Motivation in Narrative-Centered Learning Environments, Proceedings of the Workshop on Narrative Learning Environments, 13th AIED Conference, pp. 40-49, Marina del Rey, CA, July 2007.

[55] Si M., Marsella S., Pynadath D.. "Thespian: Modeling Socially Normative Behavior in a Decision-Theoretic Framework", Conference on Intelligent Virtual Agents. 2006.

[56] Si, M., Marsella, S.C., and Pynadath, D.V. 2005. THESPIAN: An Architecture for Interactive Pedagogical Drama. In AIED 2005.

[57] Aylett, R., Louchart, S., Tychsen, A., Hitchens, M., Figueiredo, R., and Mata, C. D. 2007. Managing emergent character-based narrative. In Proceedings of the 2nd international Conference on intelligent Technologies For interactive Entertainment, Cancun, Mexico, January, 2008.

[58] Dias, J and Paiva, A. Feeling and Reasoning: a Computational Model. 12th Portuguese Conference on Artificial Intelligence, EPIA (2005). Springer

[59] Jepp, P., Fradinho, M., and Pereira, J. M., An Agent Framework for a Modular Serious Game. In Proceedings of the 2010 Second international Conference on Games and Virtual Worlds For Serious Applications, March, 2010.

[60] Ortony,A., Clore G.L., Collins A., The Cognitive Structure of Emotions. Cambridge University Press, 1990, British Journal of Educational Technology.

[61] Riedl M., A comparison of interactive narrative system approaches using human improvisational actors, International Con- 
ference On The Foundations Of Digital Games, Monterey, California, 2010.

[62] Rebolledo-Mendez G., de Freitas S., Garcia Gaona A. R., "A Model of Motivation Based on Empathy for AI-Driven Avatars in Virtual Worlds", vs-games, Conference in Games and Virtual Worlds for Serious Applications, 2009Enhanced Learning Unlimited (ELU) project. Retrieved March, 2008, from http://www.eluproject.com.

[63] Lester, J.C., Towns, S.G., Callaway C.B., Voerman J.L., and Fitzgerald P.J... Deictic and Emotive Communication in Animated Pedagogical Agents, in Embodied Conversational Agents, J. Cassell, et al., Editors. 2000, MIT Press: Boston.

[64] Gatzidis, C., Parry, K., Kavanagh, E., Wilding, A., and Gibson, D., Towards the Development of an Interactive 3D Coach Training Serious Game. In Proceedings of the 2009 Conference in Games and Virtual Worlds For Serious Applications, March, 2009. doi:10.1109/VS-GAMES.2009.28

[65] Hudlicka, E., Affective game engines: motivation and requirements. In Proceedings of the 4th international Conference on Foundations of Digital Games, Orlando, USA, April , 2009.

[66] Dormann, C. and Biddle, R. 2008. Understanding game design for affective learning. In Proceedings of the 2008 Conference on Future Play: Research, Play, Share, Toronto, Canada, November, 2008.

[67] Castellano, G., Pereira, A., Leite, I., Paiva, A., and McOwan, P. W. Detecting user engagement with a robot companion using task and social interaction-based features. In Proceedings of the 2009 international Conference on Multimodal interfaces, Cambridge, Ma, USA, November, 2009.

[68] Losh, E. The palace of memory: virtual tourism and tours of duty in Tactical Iraqi and Virtual Iraq. In Proceedings of the 2006 international Conference on Game Research and Development (Perth, Australia, December 04 - 06, 2006.

[69] Johnson W. L., Developing intercultural competence through videogames, International Workshop on Intercultural Collaboration, Palo Alto, Ca, 2009

[70] Mascarenhas, S., Dias, J., Afonso, N., Enz, S., and Paiva, A. 2009. Using rituals to express cultural differences in synthetic characters. In Proceedings of the 8th international Conference on Autonomous Agents and Multiagent Systems, Budapest, Hungary, May , 2009.

[71] Doucet, L. and Srinivasan, V. 2010. Designing entertaining educational games using procedural rhetoric: a case study. In Proceedings of the 5th ACM SIGGRAPH Symposium on Video Games, Los Angeles, Ca, July, 2010.

[72] Bogost, I. Persuasive games: The expressive power of videogames. The MIT Press, Cambridge, MA, 2007.

[73] Bogost, I.. The rhetoric of video games. In The Ecology of Games: Connecting Youth, Games and Learning, K. Salen, Ed., The John D. and Catherine T. MacArthur Foundation Series on Digital Media and Learning. The MIT Press, Cambridge, MA, 2008.

[74] Swarz, J., Ousley, A., Magro, A., Rienzo, M., Burns, D., Lindsey, A.M., Wilburn, B., Bolcar, S., "CancerSpace: A Simulation-Based Game for Improving Cancer-Screening Rates," Computer Graphics and Applications, IEEE, vol.30, no.1, pp.90-94, Jan.-Feb. 2010. doi:10.1109/MCG.2010.4

[75] Doucet, L. and Srinivasan, V. 2010. Designing entertaining educational games using procedural rhetoric: a case study. In Proceedings of the 5th ACM SIGGRAPH Symposium on Video Games, Los Angeles, Ca, July, 2010.

[76] Bogost, I. Persuasive games: The expressive power of videogames. The MIT Press, Cambridge, MA, 2007.

[77] Bogost, I.. The rhetoric of video games. In The Ecology of Games: Connecting Youth, Games and Learning, K. Salen, Ed., The John D. and Catherine T. MacArthur Foundation Series on Digital Media and Learning. The MIT Press, Cambridge, MA, 2008.

[78] Swarz, J., Ousley, A., Magro, A., Rienzo, M., Burns, D., Lindsey, A.M., Wilburn, B., Bolcar, S., "CancerSpace: A Simulation-Based Game for Improving Cancer-Screening Rates," Computer Graphics and Applications, IEEE, vol.30, no.1, pp.90-94, Jan.-Feb. 2010. doi:10.1109/MCG.2010.4

[79] Doucet, L. and Srinivasan, V. 2010. Designing entertaining educational games using procedural rhetoric: a case study. In Proceed- ings of the 5th ACM SIGGRAPH Symposium on Video Games, Los Angeles, Ca, July, 2010.

[80] Bogost, I. Persuasive games: The expressive power of videogames. The MIT Press, Cambridge, MA, 2007.

[81] Bogost, I.. The rhetoric of video games. In The Ecology of Games: Connecting Youth, Games and Learning, K. Salen, Ed., The John D. and Catherine T. MacArthur Foundation Series on Digital Media and Learning. The MIT Press, Cambridge, MA, 2008.

[82] Swarz, J., Ousley, A., Magro, A., Rienzo, M., Burns, D., Lindsey, A.M., Wilburn, B., Bolcar, S., "CancerSpace: A Simulation-Based Game for Improving Cancer-Screening Rates," Computer Graphics and Applications, IEEE , vol.30, no.1, pp.90-94, Jan.-Feb. 2010. doi:10.1109/MCG.2010.4

[83] Alankus, G. M. May, A. Lazar, C. Kelleher. Towards Customizable Games for Stroke Rehabilitation. Proceedings of CHI 2010, Atlanta, GA, USA, 2010.

[84] van der Spek, E.D.; van Oostendorp, H.; Wouters, P.; Aarnoudse, L.; , "Attentional Cueing in Serious Games," 2nd International Conference on Games and Virtual Worlds for Serious Applications (VS-GAMES), March 2010. doi:10.1109/VS-GAMES. 2010.8

[85] Shabanah S. and Chen J. X., "Simplifying algorithm learning using serious games," in WCCCE '09: Proceedings of the 14th Western Canadian Conference on Computing Education. Burnaby, BC, Canada, May, 2009.

[86] Connolly, T.M., Boyle, E.A., Stansfield, M.H., and Hainey, T., "The Potential of Online Games as a Collaborative Learning Environment”, Journal of Advanced Technology for Learning, 2007

[87] McLaughlin, T., Smith, D., and Brown, I. A. 2010. A framework for evidence based visual style development for serious games. In Proceedings of the Fifth international Conference on the Foundations of Digital Games, Monterey, CA, USA, June, 2010.

[88] Pinelle, D., Wong, N., Stach, T., Heuristic Evaluation for Games: Usability Principles for Video Game Design. In Proceedings of the ACM Conference on Human Factors in Computing Systems (CHI 2008), Florence, Italy.

[89] Bellotti F., Berta R., De Gloria A., Primavera L., "Enhancing the Educational Value of Video Games”, ACM Computers in Entertainment, Vol. 7, No.2, pp. 23-41, 2009.

[90] Torrente, J., del Blanco, Á., Moreno-Ger, P., Martínez-Ortiz, I., and Fernández-Manjón, B. Implementing accessibility in educational videogames with <e-Adventure>. In Proceedings of the First ACM international Workshop on Multimedia Technologies For Distance Learning, Beijing, China, October 23 - 23, 2009

[91] Grammenos D., Savidis A., and Stephanidis C.. Designing universally accessible games. ACM Computers in Entertainment, 7(1):129, 2009. doi:10.1145/1486508.1486516

[92] Ashfield S., Jarvis C., Kaduk J., "Serious Games for Geographical Field Skills: An Exploration," vs-games, International Conference on Games and Virtual Worlds for Serious Applications, 2010. doi:10.1109/VS-GAMES.2010.18

[93] Carruthers, M. The Book of Memory. Cambridge University Press. 1990

[94] Carruthers, M. and Ziolkowski, J. The Medieval Craft of Memory: an anthology of texts and pictures. University of Pennsylvania Press. 2002

[95] Dudai, Y. Memory from A to Z. Oxford University Press. 2002.

[96] Yates, F. The Art of Memory. Trafalger Square. 1994.

[97] Squire, K. Open-ended video games: A model for developing learning for the interactive age. In The Ecology of Games: Connecting Youth, Games, and Learning, K. Salen (ed.), MIT Press, Cambridge, MA, USA. 2008.

[98] Kelly H., Howell K., Glinert E., Holding L., Swain C., Burrowbridge A., and Roper M., "How to build serious games," Commun. ACM, vol. 50, no. 7, pp. 44-49, Jul. 2007. doi: $10.1145 / 1272516.1272538$

[99] Sliney A., Murphy D., "JDoc: A Serious Game for Medical Learning," in Proc. International Conference on Advances in ComputerHuman Interaction, pp. 131-136, 2008. doi:10.1109/ ACHI.2008.50

[100]Parker J.R., Sonny Chan and Jeff Howell, "OceanQuest: A University-Based Serious Game Project”, in Proc. Digital Games Re- 
search Association Conference (DiGRA 2005),Vancouver, BC, June 16-20, 2005.

[101]Slater, M., Khanna, P., Mortensen, J., Yu, I., Visual Realism Enhances Realistic Response in an Immersive Virtual Environment. IEEE Computer Graphics and Applications, vol. 29, no. 3, 2009. doi:10.1109/MCG.2009.55

[102]Willis, J. A framework for task-based learning. Harlow,U.K., Longman Addison- Wesley, 1996

[103]Ellis, R. Task-based language learning and teaching. New York: Oxford University Press, 2003

[104]Frazer A., Argles D., and Wills G., "Is Less Actually More? The Usefulness of Educational Mini-game", in Proc IEEE International Conference on Advanced Learning Technologies, pp. 533-537, 2007

[105]Federation of American Scientists, Learning Science and Technology R\&D Roadmap. Tech. Rep., Washington D.C., Oct 2003, accepted: www.fas.org/learningfederation

[106]Moshirnia A, The Educational Potential of Modified Video Games, Informing Science and Information Technology, 2007

[107]Kuchar O. A., Huston K. and Greitzer F. L., Applying Principles of Gaming, Motivation and Learning to Enhance the effectiveness of CyberCIEGE, Report PNNL-15802, Pacific Northwest National Laboratory Richland, WA, 2006

[108]Vygotsky L., "Play and its role in the mental development of the child”. In J. Bruner, A. Jolly, \& K. Sylva, (Eds.) Play- Its Role in Development and Evolution, pp. 537-554. New York: Basic Books. 1976

[109] Bellotti F., Berta R., De Gloria A., Primavera L., "Supporting authors in the development of Task-Based Learning in Serious Virtual Worlds", British Journal of Education and Technologies
(BJET), Vol. 41, No. 1, January 2010. doi:10.1111/j.14678535.2009.01039.x

\section{AUTHORS}

Francesco Bellotti is assistant professor with the ELIOS Lab, Department of Biophysical and Electronic Engineering (DIBE), University of Genoa (e-mail: franz@elios.unige.it).

Riccardo Berta is researcher with the ELIOS Lab, Department of Biophysical and Electronic Engineering (DIBE), University of Genoa (e-mail: berta@elios.unige.it).

Alessandro De Gloria is full professor and director of teaching of the Electronic Engineering BSc and MSc at the Engineering Faculty of Genoa. He is with the ELIOS Lab, Department of Biophysical and Electronic Engineering (DIBE), University of Genoa (e-mail: adg@elios.unige.it).

This paper is an extended version of a presentation given during the "Creative Learning with Serious Games" workshop during the "Fun and Games 2010" conference in Leuwen on 15 September 2010, and was supported by the e-VITA European Life Experiences", project $\mathrm{nr}$ 143526-LLP-1-2008-1-IT-KA3-KA3MP.

Submitted October $31^{\text {st }}$, 2010. Published as resubmitted by the authors November $11^{\text {th }}, 2010$. 